\title{
On the cumulative distribution of the lithospheric plates by their areas
}

\author{
G. S. Golitsyn ${ }^{1}$ \\ Received 12 October 2017; accepted 30 November 2017; published 15 December 2017.
}

The distribution of lithospheric plates by their areas explained as the random walks in the six-dimensions space (coordinates and velocities) by Kolmogorov in 1934. KEYWORDS: size distribution of plates; random walks lows by Kolmogorov.

Citation: Golitsyn, G. S. (2017), On the cumulative distribution of the lithospheric plates by their areas, Russ. J. Earth. Sci., 17, ES5001, doi:10.2205/2017ES000607.

\section{Introduction}

One of the most astonishing laws of Nature was established by $P$. Bird 2003. He found that the cumulative number $(N)$ of the lithospheric plates in dependence on their areas $(S)$ is described by

$$
N(\geq S)=7 S^{-n}, n=0.33 .
$$

From the distribution he excluded Pacific and continental plates Africa, Antarctica, North America, Eurasia and Australia; South American plate is in the distribution. Four smallest plates like Manus are also out of it. According to Barenblatt 2003 the dependence in consideration may be called an intermediate asymptotic for about 40 plates left out of 52 considered by Bird 2003.

Such a dependence did not give a rest for this author during several years and he did not left attempts to find a simple explanation in the hope that the equation (1) is a manifestation of some deep and general science. An explanation was found at the end [Golitsyn, 2008] with the help of the theory of similarity and dimension. In that case it was supposed that a number of plates we see after the break up of Gondvana is due to the mantle convection. The mantle convection causes the geodynamics, the earthquakes arising mostly at the boundaries of the plates, the consequent quakes support the faults etc. The intensity of the convection can be described by the chief single parameter: the rate of generation of its kinetic energy per unit mass $\varepsilon$. Its mean rate is of order $10^{-11} \mathrm{~m}^{2} \mathrm{~s}^{-3}$ [Golitsyn, 2007] as a drive for geodynamics and seismicity. The value of $\varepsilon$ was chosen later as a governing parameter of the plate formation and $\varepsilon$ and area $S$ give for the cumulative number of plates which has the dimension of frequency or inverse time

\footnotetext{
${ }^{1}$ A. M. Obukhov Institute of Atmospheric Physics, RAS, Moscow, Russia

Copyright 2017 by the Geophysical Center RAS.

http://elpub.wdcb.ru/journals/rjes/doi/2017ES000607-res.html
}

$$
N(\geq S)=A(\varepsilon / S)^{1 / 3} .
$$

An important moment in these arguments is that the cumulative distribution has the dimension of frequency and its inverse value is called the mean expectation time for an event with size $\geq S$ to happen [Feller, 1968] when one deals with empirical non-normalized probabilities called histograms.

\section{Discussion}

Unfortunately the determination in the value of coefficient $A$ in 22 has been done erroneously in 2008, and also the choice of the governing parameters may be always in doubt especially without any underlying model. Therefore we decide to return to the problem. The problem evidently belongs to the class of stochastic processes developing under the action of multitude of random forces. The initial idea was proposed by $A . N$. Kolmogorov 1934. In other words the forcings are $\delta(t)$-correlated. For such a description he has proposed to use the Fokker-Plank equation

$$
\frac{\partial P}{\partial t}+u \frac{\partial P}{\partial r}=\frac{\varepsilon}{2} \frac{\partial^{2} P}{\partial u^{2}}
$$

where $P(r, u, t)$ - is the probability density for a fluid particle with velocity $u$ to be in the coordinate $r$ in the time moment $t ; \varepsilon$ is the rate of the generation (dissipation) of the particle kinetic energy (per unit mass). This equation was solved by Gledzer and Golitsyn 2010] at the initial condition $P(r, u, t=0)=\delta(u) \delta(r)$ i.e. with delta-like conditions.

The solution of eq. 2 can be presented in two forms:

$$
\begin{aligned}
P(r, u, t) & =G(u, \varepsilon t) G\left(r-\frac{u t}{2}, \frac{\varepsilon t^{3}}{12}\right), \\
P(r, u, t) & =G\left(r, \frac{\varepsilon t^{3}}{3}\right) G\left(u-\frac{3 r}{2 t}, \frac{\varepsilon t}{4}\right), \\
G(x, d) & =\frac{1}{(2 \pi d)^{1 / 2}} \exp \left(\frac{-x^{2}}{2 d}\right),
\end{aligned}
$$


In order to obtain the distribution for velocities $P(u, t)$ for arbitrary coordinates one should integrate (4) by $r$ from $-\infty$ to $\infty$. Distribution on coordinates at any velocities is obtained by integration of (4) over velocities. However the solutions will be for an infinite ensemble. However it is always necessary to know how fast solutions for finite ensemble approach to that ones for the infinite ensemble. For this Gledzer and Golitsyn 2010 integrate equations

$$
\dot{u}_{i}=a_{i}, \dot{x}_{i}=u_{i},
$$

where $i=1,2, \ldots N$ - the general number of couples of such equations at $a_{i}$ random $\delta$-like accelerations which are modeling the energy input to our system. In this case for $N \rightarrow \infty$ the second moments for velocities and coordinates are calculated as

$$
\begin{gathered}
\left\langle u^{2}(t)\right\rangle=\varepsilon t, \\
\left\langle x^{2}(t)\right\rangle=\frac{1}{3} \varepsilon t^{3} .
\end{gathered}
$$

The second moments for velocities are similar to the second moment for coordinate shift in the theory of Brownian motion and called the random walk in the coordinate space: $\left\langle\Delta x^{2}(t)\right\rangle=2 n D t$ with $D$ the diffusion coefficient and $n$ is the space dimension. Here eq. (3) describes the diffusion in the velocity space with $\varepsilon$ as the diffusion coefficient in that space [Obukhov,1959]. The formula (8) multiplied by $m / 2$ justifies the proportionality of energy on time, usually obtained by dimensional analysis as was first proposed for lagrangian fluid particle in 1944 by Landau (see Monin and Yaglom, 1975).

For us here more interesting is eq. (9). We equate rms shift of coordinate square to the area $S$ covered during random walk in the velocity space, as was first done by Batchelor 1950 for atmospheric turbulence: $S=\varepsilon t^{3}$. With such argumentation the time dependence

$$
S(t)=\frac{a}{3},
$$

where $a$ is a numerical coefficient representing, say, empty parts of the area, covered during random walk in the velocity space. Then use the definition of the cumulative distribution of probabilities as the frequency or inverse time of expectation to get an object with size, $S$ in our case, use the time from 10 and obtain

$$
N(\geq S)=\left(\frac{a \varepsilon}{3 S}\right)^{1 / 3}
$$

Golitsyn 2007] has estimated the rate of generation of kinetic energy $\varepsilon$ driving the geotectonics and seismicity as $10^{-11} \mathrm{~m}^{2} \mathrm{~s}^{-3}$. With this value converting $\mathrm{m}^{2}$ into steradian for our planet we get $\varepsilon=406 \mathrm{str} / \mathrm{s}^{3}$. Comparing 11 with (1) we obtain $7=\left(\frac{a \varepsilon}{3}\right)^{1 / 3}$, wherefrom $a=2.5$. Due to the cubic root from $(a \varepsilon)$ eq. 11 depends only weakly from both $a$ and $\varepsilon$. At this moment it is proper to recall Albert Einstein who said in 2011, as quoted by P. Bridgman 1922: right formulas obtained by dimensional analysis should not have very large or very small numerical coefficient when compared to experimental values. There is no prove of this idea but our case with finding that the numerical coefficient $a$ is of 2.5 supports well the intuition of Einstein expressed over a century ago. The combination of the solutions of the FPO equation (3) allows one also to obtain the time structure function for the velocity $u(r, t)$ proportional to time $t$ and expressing it from $(9)$ to obtain the Kolmogorov-Obukhov expressions for the small-scale turbulence in the inertial interval, also for the Richardson-Obukhov law for the turbulent diffusion expressing $K=d\left\langle x^{2}\right\rangle / d t$ according to G. I. Taylor 1915. The last deserves a special consideration. Nevertheless, all this is the consequence of the probability theory at the hypothesis on the markovian character of forcings on the system without viscous dissipation, which reveals the scale $(9)$.

Acknowledgment. I am very grateful to V. A. Kossobokov who have sent me the paper by Bird, [2003] soon after its publications and to V. F. Pisarenko who was explaining me various aspects of statistics and seismology.

\section{References}

Barenblatt, G. I. (2003), University Press, Cambridge.

Batchelor, G. K. (1950), The application of the similarity theory of turbulence to atmospheric diffusion, Quarterly Journal of the Royal Meteorological Society, 76, No. 328, 133-146, doi:10.1002/qj.49707632804

Bird, P. (2003), An updated digital model of plate boundaries, Geochem. Geophys. Geosyst., 4, No. 3, 1-52, doi:10.1029/2001GC000252

Bridgman, P. (1922), Dimensional Analysis. 1st ed, 2nd ed. 1931, Yale Univ. Press, New Haven.

Feller, W. (1968), Introduction into the Theory of Probability and its Application, 1, 528 pp., John Wiley and Sons, N.-Y..

Gledzer, E. B., G. S. Golitsyn (2010), Scaling and finite ensembles of particles in motion with the energy influx, Doklady Physics, 433, No. 8, 369-373, doi:10.1134/s102833581008001x

Golitsyn, G. S. (2007), Energy cycle of geodynamics and seismic process, Izv., Phys. Solid Earth, 43, No. 6, 443-446, doi:10.1134/S1069351307060018

Golitsyn, G. S. (2008), Size distribution of the number of lithospheric plates, Izv., Phys. Solid Earth, 44, No. 3, 175-180, doi:10.1134/S1069351308030014

Kolmogorov, A. N. (1934), Zufallige Bewegungen (Zur Theorie der Brownschen Bewegung), Annals of Mathematics, 35, No. 1, 116-117, doi:10.2307/1968123

Monin, A. S., A. M. Yaglom (1975), Statistical fluid mechanics: Mechanics of turbulence, 874 pp., MIT Press, Cambridge, Massachusetts.

Obukhov, A. M. (1959), Description of turbulence in terms of lagrangian variables, Advances in Geophysics, 6, 113, doi:10.1016/S0065-2687(08)60098-9

Taylor, G. I. (1915), I. Eddy motion in the atmosphere, Philos. Trans. R. Soc. Lond. A., 215, No. 523-37, 1-26, doi:10.1098/rsta.1915.0001

G. S. Golitsyn, A. M. Obukhov Institute of Atmospheric Physics, RAS, Moscow, Russia. (gsg@ifaran.ru) 